\title{
A SEQUENTIAL NEIGHBOR EXPLORATORY EXPERIMENTAL DESIGN METHOD FOR COMPLEX SIMULATION METAMODELING
}

\author{
Yonglin Lei \\ Wei Dong \\ Zhi Zhu \\ School of Systems Engineering \\ National University of Defense Technology \\ 109 Deya Road, Changsha, Hunan, China \\ \{yllei,dongwei,zhuzhi\}@nudt.edu.cn
}

\author{
Hessam Sarjoughian
}

Arizona Center for Integrative M\&S

Schl. of Comp., Infor, and Deci. Syst. Engr.

Arizona State University

699 S Mill Ave, Tempe, AZ 85281, USA

Sarjoughian@asu.edu

\begin{abstract}
Since complex simulation contains many factors and responses that interact in a nonlinear manner, it is important to use metamodeling for representing the causal relationships within the simulation models in a compact way. With the selected mathematical structure, the effectiveness of metamodeling depends on the comprehensiveness of the training data that is closely related to the size of the scenario space and available computing resources. Generally, sequential experimental design methods are more efficient than one-shot ones, but the later depend on the domain knowledge of the experimenters and are uneasy to be conducted automatically. This paper proposes a sequential neighbor exploratory experimental design (SNEED) method for metamodeling purpose. Through the peaks function example, we compare this new method to Latin hypercube with a support vector regression metamodel trained by their training data respectively. The result shows that under the same experiment sample count, the SNEED method produces better regression performance.
\end{abstract}

Keywords: Simulation metamodel, sequential experimental design, neighbor exploratory, Latin Hypercube, k-d tree.

\section{INTRODUCTION}

The scenario space of complex simulation is huge because it usually contains a number of factors that influence a number of responses in a nonlinear manner. In order to avoid the problem of dimension explosion, metamodeling plays a key role in the evaluation and optimization of complex system design alternatives based on simulation. In general, the process of simulation metamodeling involves six principal steps: basic scenario preparation, factors screening, simulation experimental design, simulation experiment running, metamodel training, and metamodel validation. Significantly, the selection of an effective simulation experimental design method is closely related to the computation complexity of the experiment and the validity of the metamodel, determining the ultimate success of a simulation metamodeling effort.

Currently, the simulation experimental design method can be roughly divided into non-sequential and sequential categories according to whether or not incorporating the execution of the simulation under investigation. Non-sequential methods are one-shot design without taking system responses into consideration. Classical DOE (Design of Experiment) methods, like factorial design, orthogonal array 
(OA), Latin hypercube sampling (LHS), and central composite design (CCD) are all non-sequential. These methods are all generic and can be applied in any given system without dedicating to simulation metamodeling.

Unlike non-sequential methods, sequential methods view simulation experimental design as a process with a number of iterations, each of which is required to execute the simulation to get the experimental data. By analyzing the experimental data, one can know the region that is needed to be sampled in the next round of iteration and continue the iterating process. In general, there are two kinds of sequential methods: optimization-oriented and metamodeling-oriented. Although both are trying to reduce the number of experiments, their goals are different. The former tends to find the global optimization, and the latter tries to get the global approximation of the simulation. This research focuses on the metamodeling-oriented experimental design method with better sampling efficiency.

Traditionally, metamodeling-oriented sequential methods are related to the regression model selected, and depends on the domain knowledge of the system under investigation as well as manual intervention. As a result, this kind of experiments are difficult to be conducted automatically. There are needs for a sequential method that is able to tackle with different kinds of regression models and target systems in an adaptive manner. Related researches are investigated when proposing such a method. Simulation or computer experiment oriented experimental design is distinguished from classical DOE under the cover of modern DOE methods. (Simpson et al. 2002; Giunta et al. 2003; and Kleijnen 2005) provide some surveys of modern methods. Alam et al. (2004) and Alam et al. (2006) discussed the non-sequential experimental design for simulation metamodeling with incorporation of domain knowledge. Donohue et al. (1993) and Rossetti et al. (2009) stated a collection of issues about the simulation metamodeling design based on the sequential method. Zhu (2016) proposed a gradient-based method for sequential sampling. A couple of sequential methods that are able to perform the experiment design, analysis, and metamodeling in an adaptive or automatic way are proposed based on distributed or supercomputers (Gramacy and Lee 2006; Gramacy and Lee 2009; Gorissen et al. 2006).

Considering the requirements of complex simulation metamodeling, this paper proposes a sequential neighbor exploratory experimental design method. This new sequential method employs a combination of three sampling strategies: the quasi gradient-based sampling, random neighbor exploratory sampling, and polynomial regression metamodel sampling. Quasi gradient-based sampling is employed to exploit domain knowledge. Random neighbor exploratory sampling are adopted for generalization purpose. For place where exists weak nonlinear characteristic, a polynomial regression based metamodel is generated and sampled to avoid the problem of over-fitness in the global metamodel. Compared with the traditional methods, it has some advantages. The non-linear areas can be adaptively sampled with more points and represented as accurately as necessary. The simulation metamodel is only trained only one time instead of many times as in other sequential methods. It has better generality for not depending on experimenters' domain knowledge and shows substantial potentials towards automatic sequential experiment and simulation metamodeling.

\section{SEQUENTIAL NEIGHBOR EXPLORATION EXPERIMENTAL DESIGN METHOD}

The flowchart of abovementioned six-step simulation metamodeling is shown in the left part of Figure 1. For sequential experimental design methods, Step C (simulation experimental design) and Step D (simulation experiment running) are usually contained within an multi-round iterative process. For the new proposed Sequential Neighbor Exploration Experimental Design (SNEED), this process is divided into a five-step iterative process as shown in the right part of Figure 1. 


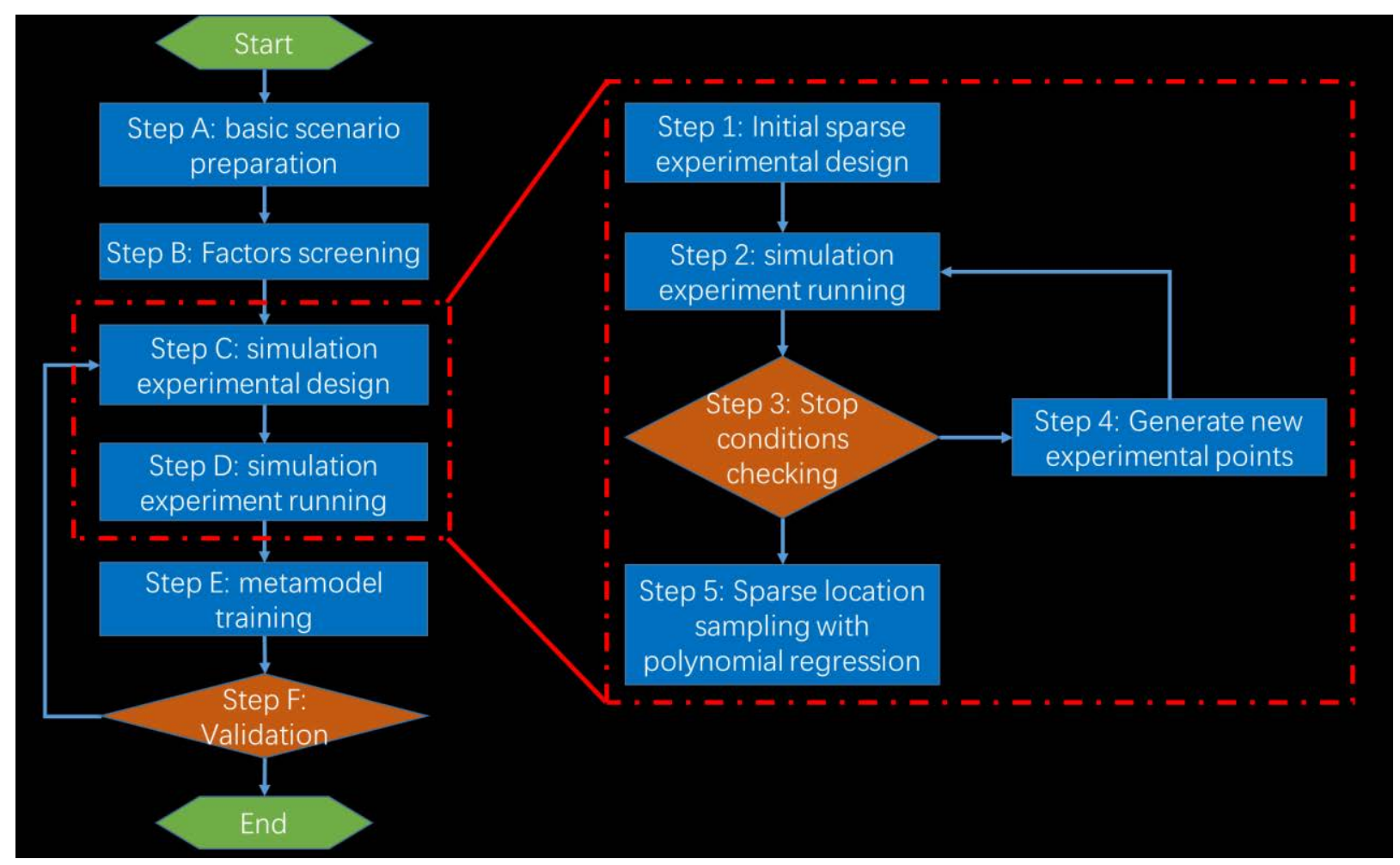

Figure 1: The flowchart of simulation metamodeling (left) based on the SNEED method (right).

\section{Step1: Initial sparse experimental design}

For generality, we can safely assume the system to be simulated is completely unknown at start. A traditional experiment design method, factorial design, uniform design or LHS, is used to generate a small number of initial experimental points with the objective to make a preliminary detection of the system. In general, the full factorial design is preferred since it is the most robust choice. For LHS there should be a little more experimental points than normal to ensure that the sensitive areas of the system are more easily found so as to ensure the stability of the algorithm, especially for those systems most of whose responses are not sensitive to input factors and the size of their sensitive areas are small. In practice, 100 to 200 experimental points are suggested.

\section{Step2: Simulation experimental running}

Given those experimental design points, the second step is to conduct simulation experimental running and collect experimental data. In order to make this experimental design method general and reliable, it is necessary to process both input factors and output experimental data by ways of suitable operations like logarithm or normalization. The inputs and outputs of a simulation experiment are generally dimensional but the experimental points need to be evenly distributed and their values should be dimensionless. The purpose of data processing is to ensure the generality of the algorithms in different systems.

\section{Step3: Stop conditions checking}

The main stop condition is satisfied when the number of points generated by a single iteration is smaller than a certain fraction of the number of generated points. The rational here is that if the number of new experimental points generated by this iteration is less than a certain threshold ratio of the total number of generated experimental points, then it can be considered that further iteration cannot significantly discover more system knowledge. One additional condition can also be set to avoid too many iterations and computation resources consumption by a maximum number of generated experimental points or maximum number of iterations. 


\section{Step4: Generating randomly new experimental points}

The strategy to generate new experimental points is the key step of this new method. The basic idea is to iteratively explore all neighbor points for each experimental point to check whether or not to generate a new experimental point according to a conception of quasi-gradient. Here, we don't adopt a threshold-based method, since threshold setting is specific to the system under investigation, and will greatly affect the final effect and stability of the algorithm. Firstly, the quasi-gradient between current point and each of its neighbor points is computed based on the Euclidean distance and response difference between the two experimental points. Then a probability of generation or not is computed based on the value of quasigradient. If the sample value of this probability is 1 , there will be a new experimental point generated. The location of this new point is generated randomly. For example, it can be based on a normal distribution, the mean and variance of which are determined by the current point and its neighbor in each dimension. Such randomness helps to uncover un-explored sensitive areas of the system.

\section{Step5: Sparse location sampling with polynomial regression}

When the iteration stops, the distribution of the experimental points in the sample space is determined by the characteristics of the system itself. Specifically, more experimental points are distributed in the sensitive area of the system, whereas sparse experimental points are found in the insensitive areas. These sparse experimental points are unfavorable to metamodel training since these regions are prone to over-fitting. To avoid this problem, it is necessary to increase the density of experimental points in these areas. Polynomial linear regression is used to sample and complement the experimental data of the sparse region where outputs are not sensitive to inputs.

In order to make better use of polynomial regression to fill sparse region with experimental data, a recursive binary regression algorithm is proposed. Similar to the k-d tree, this new algorithm is more intelligent, flexible, and effective than direct partitioned block regression. In this method, the recursive partitioning principle is used to achieve segmentation and regression of the entire input space. Its recursive process is as follows. Fit the input space based on a linear model of low-order polynomial. If the fitting effect meets the requirements, the experimental points are complemented directly by sampling the linear regression model. Otherwise, dividing the sample points into two parts based on the maximum dimension midpoint. In each part, the recursive binary regression algorithm is used respectively. In this way, all sparse region experimental points can be completed by calling the recursive binary regression algorithm in the entire input space.

\section{DETAILED DESIGN OF NEW EXPERIMENTAL POINTS GENERATION}

As stated earlier, the Step 4 is crucial in the proposed sequential neighbor exploratory experimental design method and deserves further discussions.

\subsection{K-d Tree-based Neighbor Experimental Points Search}

To explore all neighbor experimental points of each experimental point, one needs to traverse all simulated experimental points and search all neighbor experimental points. Let the number of all simulated experimental points be $n$, then the time complexity of traversing them one by one is $O\left(n^{2}\right)$. But using k-d tree to optimize this searching process can reduce the time complexity to $O(n \log n)$, greatly improving the efficiency of the algorithm.

A k-dimensional tree is a data structure that partitions a k-dimensional data space, which is mainly used in the search of key data in multidimensional space. In essence, $\mathrm{k}-\mathrm{d}$ tree is a balanced binary tree and also a spatially partitioned tree. $\mathrm{k}-\mathrm{d}$ tree divides the entire space into specific parts and performs search operations within a specific part. 
In general, the process of portioning k-d tree consists of two steps to find neighbors points with a total time complexity of $O(n \log n)$.

1. Building a k-d tree based on the experimental data. The time complexity is $O(n \log n)$.

2. For each point find the nearest neighbors in the k-d tree. For $n$ points, there will be $n$ search operations during the search phase. Since the k-d tree is a binary search tree, it is easy to know that the time complexity of finding the neighbor points of each point is $O(\log n)$, so the total time complexity is $O(n \log n)$.

\subsection{New Experiment Point Generation Probability Computation}

The new experimental point generation probability is computed based on a conception of quasi-gradient. The quasi-gradient is defined as:

$$
\xi_{i j}=\frac{2}{\pi} \arctan \left(\frac{\delta_{i j}}{D_{i j}}\right)
$$

where $\xi_{i j}$ is the quasi-gradient of experimental point $P_{i}$ and $P_{j}, \delta_{i j}$ and $D_{i j}$ are the normalization of the response difference and the Euclidean distance between $P_{i}$ and $P_{j}$ responsively. $\xi_{i j} \in[-1,1], \delta_{i j} \in$ $[-1,1], D_{i j} \in[-1,1]$.

The probability of generating new experimental points between two points is negatively related to the distance and positively related to the response difference. Different generation probability functions will have an influence on the final effect of the proposed method in this paper. Following is a good probability generation algorithm and its function image is shown in Figure 2.

$$
P_{g}=\frac{\left(\delta_{i j}{ }^{2}+D_{i j}{ }^{2}\right)}{2} \times \xi_{i j}{ }^{2}
$$

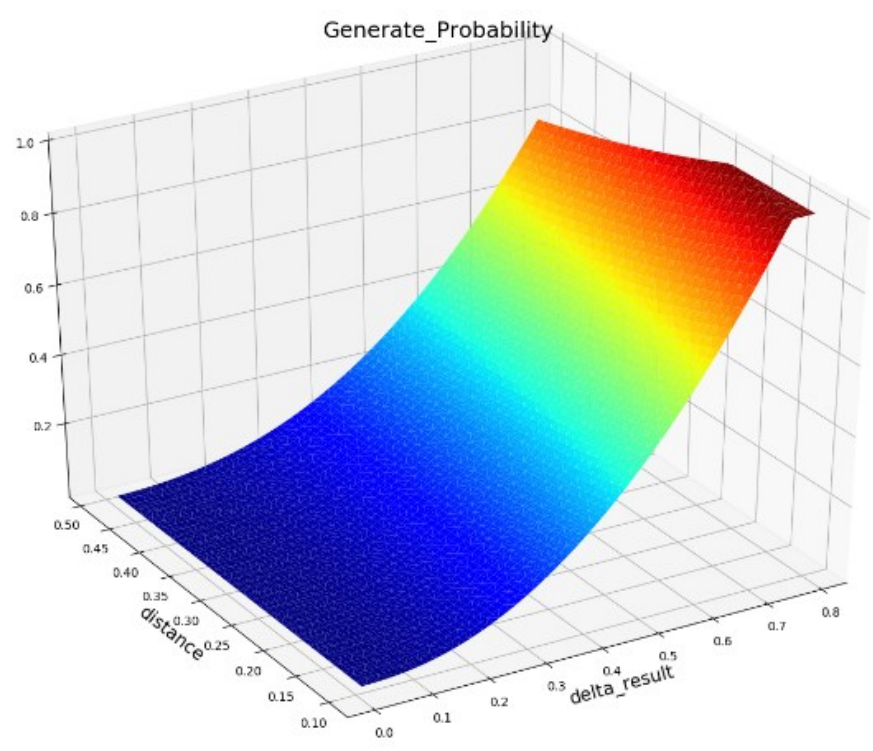

Figure 2: The function image of generating probabilistic algorithm.

\subsection{New Experimental Point Position Calculation Based on Normal Distribution}

The new experiment point will be generated in the form of normal distribution between the current experiment point and its neighbor. Furthermore, each dimension is assumed to obey a normal distribution 
with the mean being the midpoint value of the current point and its neighbor point in this dimension, and the variance is $1 / 6$ of the difference in that dimension. The new experimental point is more likely to appear in darker areas as shown in Figure 3.

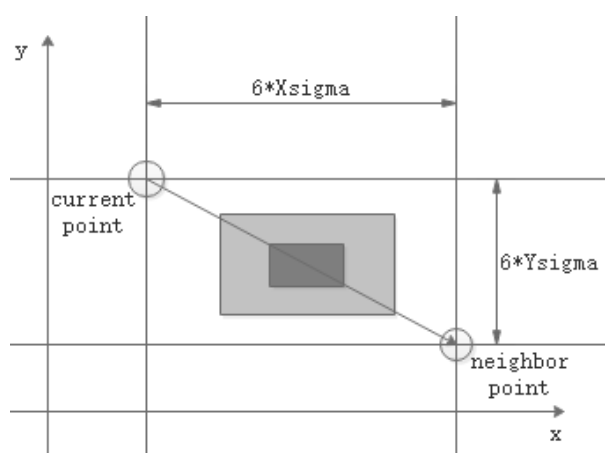

Figure 3: Occurrence probability distribution of new experimental points.

\section{CASE STUDY}

\subsection{The exemplar system: peaks function}

As an illustration, we select the peaks function (1), which is a two inputs and one output system, as the experimental target. The sensitive region of the system will be placed at a random position within the value domain to verify that the points generated by the algorithm are indeed distributed in the system's sensitive area. The height map and 3D view of the peaks function is shown in Figure 4. Note that $x \in[-10,5]$, $y \in[-5,10]$.

$$
\operatorname{peaks}(x, y)=5(1-x)^{2} e^{-x^{2}-(y+1)^{2}}-5\left(\frac{w}{5}-y^{5}\right)^{2} e^{-x^{2}-y^{2}}-6 e^{-(x+1)^{2}-y^{2}}
$$
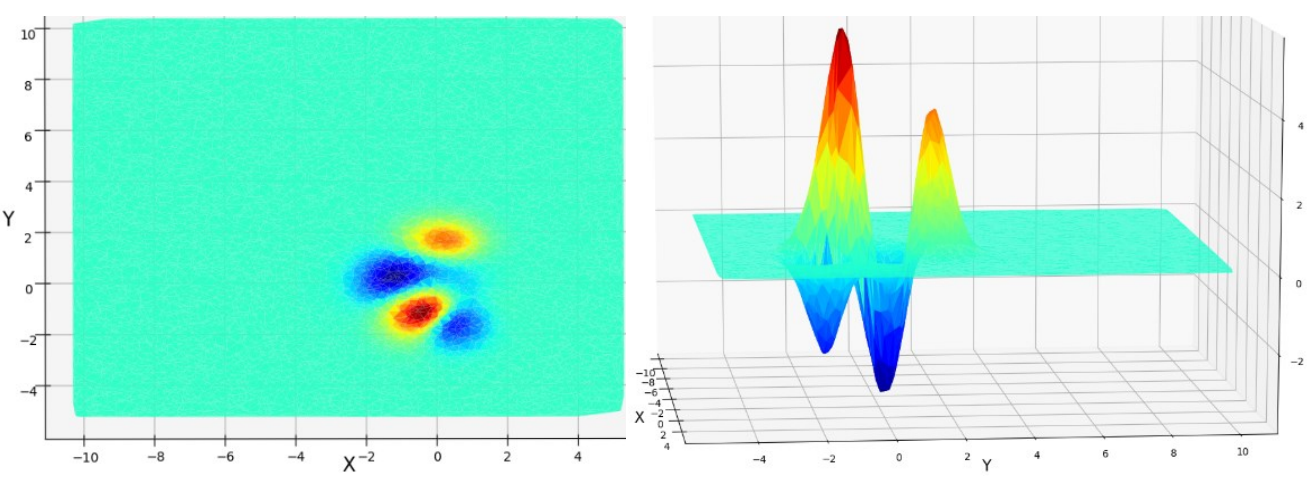

Figure 4: The peaks function height map and 3D view.

Obviously, the system represented by the peaks function is sensitive when $x \in[-3,2], y \in[-3,3]$ and is constant in other regions. However, the concrete mapping of the system described by the peaks function is still unknown. In this case, we can only roughly determine $x \in[-10,5], y \in[-5,10]$. Such a system will be sampled using the proposed SNEED method. We will show SNEED method can support the simulation metamodeling with fewer experiments (lower computation cost) and better fitting effects. The experimentation is developed with Python3.5 in Windows 10. The applied third party libraries include numpy for exemplar system computation, sklearn for SVR metamodel training, and matplotlib for plotting. 


\subsection{Sequential experimental design and sampling according to the SNEED method}

Initially, 100 points are selected in the scenario space using LHS as the initial set of experimental points. According to the method described above, stop conditions will be satisfied after three iterations. Specifically, the first iteration generates 98 sample points, the second iteration generates 134, and the third iteration generates 22 sample points that is less than $10 \%$ of the existing sample points (35), so the iteration process ends. So far, a total of 354 experiments were performed to obtain 354 points. After each new experiment point is added, the experimental points are distributed in the input space as shown in Figure 5. Comparing with Figure 4, we can find that the experimental points generated by each iteration are distributed in the sensitive area of the system.
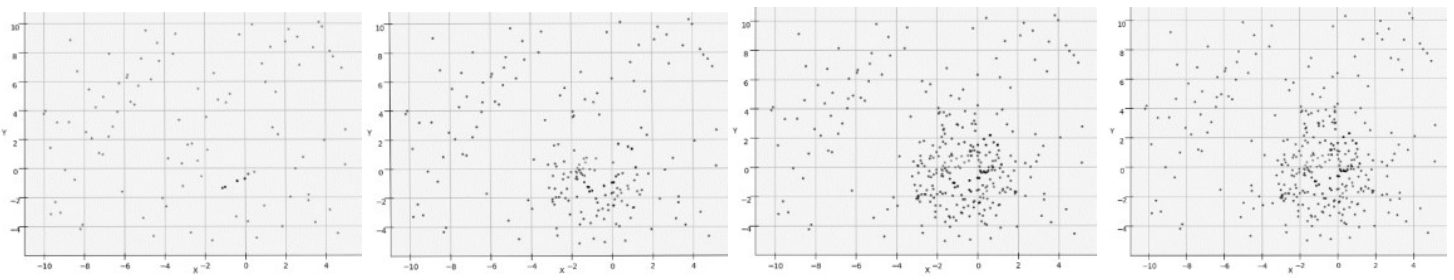

Figure 5: Distribution of experimental points obtained after each iteration.

Based on the 354 experimental points, a recursive binary regression algorithm was used to complement the sparse regions of the input space and get 3,000 training data. Comparing the training data obtained by the LHS method of the same size of trials, the training data obtained by this method is almost 9 times that of the latter, as shown in Figure 6.
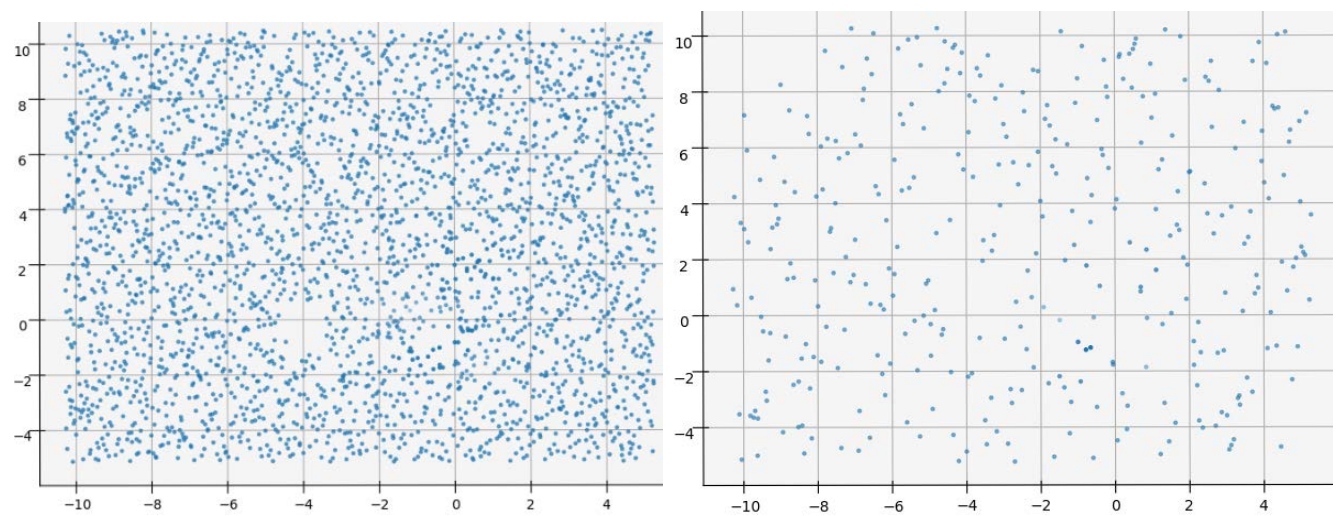

Figure 6: Training data obtained from the SNEED (left) and LHS (right).

\subsection{Metamodeling and comparison with LHS}

The system metamodel is trained using the training data obtained based on the above two methods. Using the Gaussian kernel transformation, the SVR metamodel with a gamma 1 can fit its own training data well on both training sets. Comparing the height map and the three-dimensional view of the SVR model trained on two training sets, we can find that the two peaks and valley heights based on the LHS method have large deviations from the original system, as shown in Figure 7. The SVR metamodel based on the SNEED method restores the original system features better. The reason is that the LHS method cannot fully extract the system features, so the internal information of the system caused by insufficient sampling cannot be fully reflected in the set of training data. 

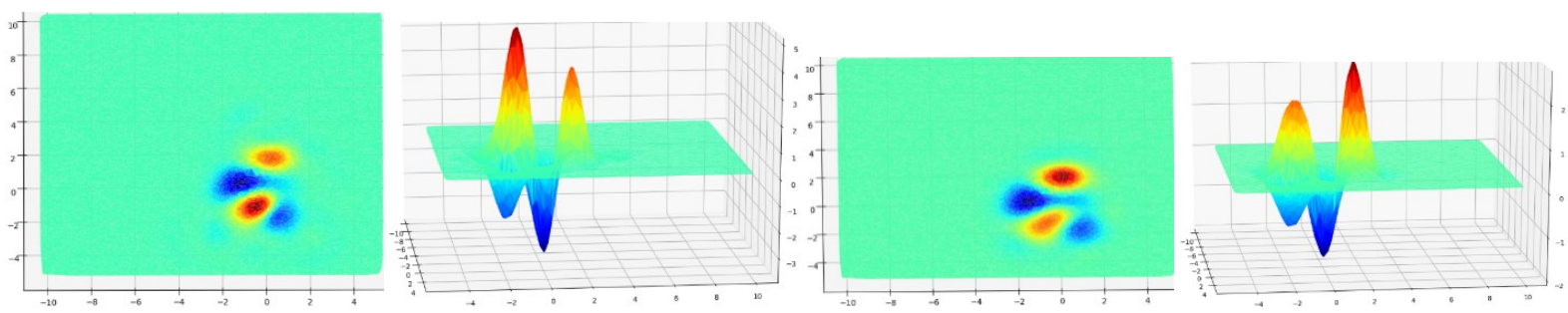

Figure 7: The height hot zone map and 3D view of support vector model that is based on the sequential neighbor exploration (left two) and the Latin hypercube sampling (right two).

Consider 1000 points from the LHS method as the test set, we select a couple of model evaluation indicators to evaluate the performance of the SVR metamodel obtained by the two methods on the test set, as shown in Table 1.

Table 1: Comparison between SNEED and LHS.

\begin{tabular}{lllll}
\hline Method NO. & Explained variance score & Mean absolute error & Mean squared error & R2 score \\
\hline 1 & 0.6925 & 0.1202 & 0.0909 & 0.6821 \\
2 & 0.9854 & 0.0712 & 0.0069 & 0.9755 \\
\hline
\end{tabular}

It can be seen that the SVR metamodel based on the SNEED method performs significantly better than the LHS on the four model evaluation indicators. With the same evaluation indicators, in addition, the two methods in five replicates is respectively shown in Table 2 and Table 3 and a comparison of visual effects is shown in Figure 8.

Table 2: Five repetitions of the LHS method.

\begin{tabular}{lllll}
\hline NO. & Explained variance score & Mean absolute error & Mean squared error & R2 score \\
\hline 1 & 0.5322 & 0.0982 & 0.1284 & 0.5169 \\
2 & 0.6715 & 0.0921 & 0.1009 & 0.6609 \\
3 & 0.6914 & 0.1346 & 0.0908 & 0.6728 \\
4 & 0.7425 & 0.1252 & 0.0716 & 0.7237 \\
5 & 0.6973 & 0.0750 & 0.0887 & 0.6973 \\
Avg. & 0.6670 & 0.1050 & 0.0961 & 0.6543 \\
\hline
\end{tabular}

Table 3: Five repetitions of the SNEED method.

\begin{tabular}{lllll}
\hline NO. & Explained variance score & Mean absolute error & Mean squared error & R2 score \\
\hline 1 & 0.9793 & 0.0862 & 0.0106 & 0.9600 \\
2 & 0.9685 & 0.0566 & 0.0097 & 0.9673 \\
3 & 0.9824 & 0.0740 & 0.0078 & 0.9716 \\
4 & 0.9845 & 0.0646 & 0.0059 & 0.9770 \\
5 & 0.9896 & 0.0825 & 0.0080 & 0.9725 \\
Avg. & 0.9809 & 0.0728 & 0.0084 & 0.9697 \\
\hline
\end{tabular}




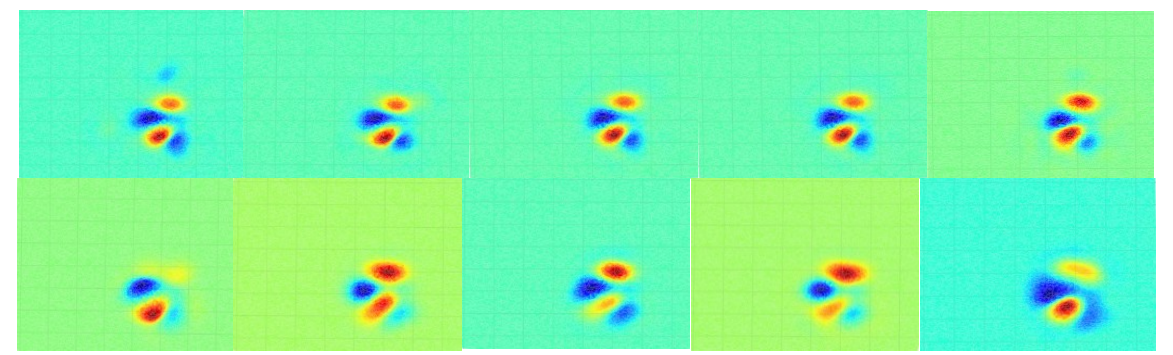

Figure 8: Comparison of the effects of SNEED (above) and LHS (below) in the five replicate experiments.

From the statistical data of repeated experiments, we can find that the SVR metamodel based on the SNEED method proposed in this paper has better model explanatory power, smaller error and stronger stability.

\section{CONCLUSIONS}

Compared with the traditional one-shot experimental design method, the proposed SNEED method has obvious advantages. It makes the simulation metamodel have better model explanatory power, smaller error and stronger stability. Compared with the LHS method with the consideration of domain knowledge, the SNEED method does not require domain knowledge and is suitable to any unknown system. The SNEED method can be used as a data acquisition method for metamodeling a nonlinear complex system. It has the advantages of rapidity, stability, and strong adaptability, and is able to improve the data collection efficiency in the process of the establishment of a simulation metamodel.

\section{REFERENCES}

Alam, F. M., K. R. McNaught, and T. J. Ringrose. 2004. "A comparison of experimental designs in the development of a neural network simulation metamodel". Simulation Modelling Practice \& Theory, vol. 12, pp. 559-578.

Alam, F. M., K. R. McNaught, and T. J. Ringrose. 2006. "An Artificial Neural Network Based Metamodel for Analysing a Stochastic Combat Simulation". International Journal of Enterprise Information Systems vol. 2, pp. 38-57.

Alaeddini, A., K. Yang, and A. Murat. 2013. "ASRSM: A Sequential Experimental Design for Response Surface Optimization”. Quality Reliability Engineering vol. 29, pp. 241-258.

Donohue, J. M., E. C. Houck, and R. H. Myers. 1993. "A Sequential Experimental Design Procedure for the Estimation of First- and Second-Order Simulation Metamodels". ACM Transactions on Modeling and Computer Simulatlon vol 3, pp. 190-224.

Giunta, A. A., S. F. Wojtkiewicz, and M. S. Eldred. 2003. "Overview of Modern Design of Experiments Methods for Computational Simulations". In Proceeding s of the 41st aerospace sciences meeting and exhibit. pp. 1-17.

Gorissen, D., K. Crombecq, W. Hendrickx, and T. Dhaene. 2006. "Adaptive Distributed Metamodeling”. In Proceedings of the 7th international conference on high performance computing for computational science, pp. 579-588.

Gramacy, R. B. and H. K. H. Lee. 2006. "Adaptive design of supercomputer experiments". http://www.soe.ucsc.edu/share/technical-reports/2006/ams2006-13.pdf. Accessed Jan. 19, 2018.

Gramacy, R. B. and H. K. H. Lee. 2009. "Adaptive design and analysis of supercomputer experiments". Technometrics vol 51, pp. 130-145.

Hendrickx, W. and T. Dhaene. 2005. "Sequential Design and Rational Metamodelling". In Proceedings of the 2005 Winter Simulation Conference, pp. 290-298. 
Kleijnen, J. P. C. 2005. "An overview of the design and analysis of simulation experiments for sensitivity analysis”. European Journal of Operational Research vol. 164, pp. 287-300.

Rossetti, M. D, R. R. Hill, and B. Johansson. 2009. "A Novel Sequential Design Strategy for Global Surrogate Modeling”. In Proceedings of the 2009 Winter Simulation Conference. pp. 731-742.

Simpson, T. W., Dennis K. J. L., and W. Chen. 2002. "Sampling Strategies for Computer Experiments: Design and Analysis". International Journal of Reliability and Applications. pp. 1-37

Zhu, R. 2016. "Gradient-based Sampling: An Adaptive Importance Sampling for Least-squares". In Proceedings of the 30th Conference on Neural Information Processing Systems, pp. 1-13.

\section{AUTHOR BIOGRAPHIES}

YONGLIN LEI is a Professor in the School of System Engineering at National University of Defense Technology. He holds a PhD in Systems Engineering from National University of Defense Technology. His research interests lie in system engineering, simulation modeling, and model-driven architecture. His email addressisyllei@nudt.edu.cn.

WEI DONG is a Master in Management Science and Engineering from National University of Defense Technology, His research interests include simulation experiment design. His email address is dongwei@nudt.edu.cn.

ZHI ZHU is an Assistant Professor in the School of System Engineering at National University of Defense Technology. He holds a Ph.D. in Military Equipment from National University of Defense Technology. His research interests include domain specific language and model-driven engineering. His email address is zhuzhi@nudt.edu.cn.

HESSAM SARJOUGHIAN is an Associate Professor of Computer Science and Computer Engineering in the School of Computing, Informatics, and Decision Systems Engineering (CIDSE) at Arizona State University (ASU), Tempe, AZ, and the co-director of the Arizona Center for Integrative Modeling \& Simulation (ACIMS). His email address is sarjoughian@asu.edu. 